\title{
Computational Asset Allocation Using One-Sided and Two-Sided Variability Measures
}

\author{
Simone Farinelli ${ }^{1}$, Damiano Rossello ${ }^{2}$, and Luisa Tibiletti ${ }^{3}$ \\ 1 Quantitative and Bond Research, Cantonal Bank of Zurich, \\ P.O. Box, CH-8010 Zurich, Switzerland \\ simone.farinelli@zkb.ch \\ 2 Department of Economics and Quantitative Methods, \\ University of Catania, Corso Italia, 55, 95129 Catania, Italy \\ rossello@unict.it \\ 3 Department of Statistics and Mathematics "Diego de Castro", \\ University of Torino, Piazza Arbarello, 8, 10122 Torino, Italy \\ luisa.tibiletti@unito.it
}

\begin{abstract}
Excluding the assumption of normality in return distributions, a general reward-risk ratio suitable to compare portfolio returns with respect to a benchmark must includes asymmetrical information on both "good" volatility (above the benchmark) and "bad" volatility (below the benchmark), with different sensitivities. Including the Farinelli-Tibiletti ratio and few other indexes recently proposed by the literature, the class of one-sided variability measures achieves the goal. We investigate the forecasting ability of eleven alternatives ratios in portfolio optimization problems. We employ data from security markets to quantify the portfolio's overperformance with respect to a given benchmark.
\end{abstract}

\section{Introduction}

Investment performance evaluation requires appropriate tools for ranking different risky projects. Nowadays most practitioners employ reward-risk indexes developed by academics. Nevertheless, only with normality assumption the uncertainty of future wealth is fully captured by the first two moments of the excess return's distribution. In presence of kurtosis and skewness, two-sided reward and risk measures, i.e. the average excess return and a dispersion measure considering both positive and negative deviations from the mean, do not separate information about overperformance and underperformance, causing an anomaly since investors typically distinguish between upside and downside risk. In recent years several alternatives to the Sharpe ratio has been proposed to avoid sub-optimal choices in portfolio selection problems. Some of these redefine the risk measure in the denominator, such as the Gini ratio [13], [16], the mean absolute deviation (MAD) ratio [8], the stable ratio [1], [7], the mini-max ratio [17], the SortinoSatchell ratio [14], [15], [12], the VaR and STARR ratios, [6], [9]. The first three refer to modified versions of the dispersion (risk) measure. The Sortino-Satchell, VaR and STARR ratios have a denominator that accounts for downside risk (the mini-max ratio is a special case of STARR). Performance indexes based on one-sided variability 
measures such as the Farinelli-Tibiletti ratio [3], [4], [5] and Rachev's ratios [1], [2] use instead an upper partial moment (deviations above the benchmark, for the reward), and a lower partial moment (deviations belove the benchmark, for the risk) of different orders. The higher the order, the higher the agent's inclination towards or dislike of the extreme events 1

The performance measures mentioned above lead to different optimal allocation. Moreover, since the joint distribution of future total returns are not known but can be only estimated, an empirical analysis is needed to exploit the ranking of alternative performance indexes. We will illustrate results from portfolio optimization problems considering five time series of total return indexes from 3 countries and employing 11 performance indexes. Historical data show high volatility affecting the joint empirical distribution of returns which is fat-tailed.

The paper is organized as follows. Section 2 reviews one-sided variability measures. Section 3 formulates the optimal portfolio problem, and contains the numerical examples and the back tests. Section 4 contains some concluding remarks.

\section{One-Sided Variability Measures}

Let $R$ be a $p$-integrable random variable representing the total return over a fixed horizon related to a certain investment. A general performance index is given by the ratio $\Phi(R):=r(R) / \rho(R)$, for the investment opportunity having reward $r(R)$ and risk $\rho(R)$. The higher $\Phi(R)$, the more preferable the risky trade $R$.

Given a benchmark 2 , the excess portfolio return writes $X=R-b$. One-sided variability measures are to be used if we want to separate information about the likelihood of a positive performance above the minimum acceptable return, $X>0$, and the likelihood of underperforming the target return, $X<0$. Recently, Farinelli and Tibiletti (see [3], [4], and [5]) have introduced a new reward-risk index based on the right-sided moment of $p$-th orden ${ }^{3} \mathrm{E}\left[\left(X^{+}\right)^{p}\right]^{1 / p}$ (used to value reward $\left.r(X)\right)$ and the left-sided moment of $q$-th order $\mathrm{E}\left[\left(X^{-}\right)^{q}\right]^{1 / q}$ (used to value risk $\rho(X)$ ). The choice of $p$ and $q$ reflects different agent's feelings about the consequences of either overperformance or underperformance. A small value for the left order, $q<1$, is appropriate if the agent is willing to fail the target without particular regard to the amount. When large deviations below $b$ harm more than the small ones, then one can chooses $q>1$. A similar reasoning applies to selecting the proper right order.

The idea behind Rachev's ratios is similar, but instead of looking at the positive or negative dispersion around the expectation, the focus is on the left and right tail of the excess portfolio return's distribution. In the Rachev's Generalized Ratio one has $r(X):=\mathrm{E}\left[\left(X^{+}\right)^{\gamma} \mid X \geq-V a R_{1-\alpha}\right]$ and $\rho(X):=\mathrm{E}\left[\left(X^{-}\right)^{\delta} \mid X \leq-V a R_{\beta}\right]$, where

\footnotetext{
${ }^{1}$ This conceptual line is similar to that of a pension fund manager's view. He synthesizes between the portfolio manager's aim (to beat the chosen benchmark, controlling upside risk) and the risk manager's aim (to control downside risk).

${ }^{2}$ The portfolio under consideration consists in a long position of the effective managed portfolio and a short position of the benchmark, such as a life/non-life insurance liability, a market index and a money market instrument.

${ }^{3}$ Here $x^{-}$denotes $-\min \{x, 0\}$ and $x^{+}$denotes $\max \{0, x\}$.
} 
$\alpha, \beta \in(0,1)$, the parameters $\gamma, \delta>0$ behave similarly to $p$ and $q$, while the Value-atRisk is defined as $\operatorname{VaR}_{c}:=-\inf \{x \mid \mathrm{P}(X \leq x)>c\}$.

\section{Back Test of Performance Indexes}

Investors wish to maximize a performance measure finding an optimal risky portfolio regarded as the "market portfolio" in which to invest their wealth. In finite dimensional setting, let $\mathbf{r}=\left(r_{1}, \ldots, r_{N}\right)^{\prime}$ be a random vector representing the total returns of $N$ assets over a fixed horizon $\square^{4}$ The optimal risky portfolio $\mathbf{w}=\left(w_{1}, \ldots, w_{N}\right)^{\prime}$ solves the static stochastic optimization problem (SSO)

$$
\begin{gathered}
\max _{\mathbf{w} \in \mathcal{W}} \Phi\left(\mathbf{r}^{\prime} \mathbf{w}\right) \\
\text { s.t. } \mathcal{W}:=\left\{\mathbf{w} \in \mathbb{R}^{N} \mid \mathbf{e}^{\prime} \mathbf{w}=0, \mathbf{l}_{\mathbf{B}} \leq \mathbf{w} \leq \mathbf{u}_{\mathbf{B}}\right\}
\end{gathered}
$$

where $\mathbf{e}$ is a vector of ones, $\mathbf{l}_{\mathbf{B}}$ and $\mathbf{u}_{\mathbf{B}}$ are the vectors of lower bounds and upper bounds for portfolio weights 5 With problem (1) in mind, we do an empirical comparison among the performance indexes in order to verify their forecasting ability when the reward measures and the risk measures used admit different definitions with respect to the traditional Sharpe ratio, particularly those ratios based on one-sided variability measures. Considering several time steps, we chose the unit time step equal to 1 month and model the re-allocation decision with a sequence of one-period SSOs 6 In the following, we refer to $\left(r_{t i}\right)_{t=-T, \ldots, 0,1 \ldots, h}$ as the time series of stock index $i$, where $T$ denotes the size of sample data, and $h$ is the time lag.

\subsection{Numerical Examples}

To analyze the behavior of the 11 performance indexes mentioned in this paper, we propose an application to stock portfolio optimization. The primary issue being optimization, we employ the Exponential Weighted Moving Average model (EWMA) for forecasting the future total returns. One expects some of the optimized portfolio will generate an overperformance 7

- Asset universe. Five stock indexes with reinvested dividend (total return indexes) from 3 countries: S\&P500 $(i=1)$, DowJones $(i=2)$, NASDAQ $(i=3)$, FTSE $(i=4)$, and NIKKEI $(i=5)$. Benchmark: T-bill as a proxy for the risk-free rate $(i=6)$. Number of assets: $N=6$.

\footnotetext{
${ }^{4}$ Assume the discrete compounding convention.

${ }^{5}$ Given the total return $R=\mathbf{r}^{\prime} \mathbf{w}_{\mathbf{p}}$, the benchmark can be represented as $b=\mathbf{r}^{\prime} \mathbf{w}_{\mathbf{b}}$. Hence, the total excess return can be represented as $X=\mathbf{r}^{\prime} \mathbf{w}$, where the difference $\mathbf{w}=\mathbf{w}_{\mathbf{p}}-\mathbf{w}_{\mathbf{b}}$ is termed as excess weights. Note that $\mathbf{w}_{\mathbf{p}}=\left(0, w_{2}, \ldots, w_{N}\right)^{\prime}$ and $\mathbf{w}_{\mathbf{b}}=(1,0, \ldots, 0)^{\prime}$, hence the benchmark's return is a component of $\mathbf{r}$.

${ }^{6}$ This choice guarantees stochastic independence over time, since the historical returns we will use are weakly stationary. Otherwise, a stochastic dynamic programming approach were more adequate, though the pseudo-dynamic optimization approach we will use is a good starting point to estimate the solution of the former.

${ }^{7}$ If that is true, it is admissible to say that a performance ratio has a forecasting power.
} 
- Historical data. A data set comprising monthly total returns and T-bill rates from April 2, 1984, to October 3, 2005, for a total of $258 \times 6$ observations 8 Note that $t=0$ corresponds to February 1, 2005 and $t=h=8$ to October 3, 2005. The initial data set contains $T=250$ observations and it is used for estimation and forecasting purposes.

- Investment inequality restrictions. Two cases considered, to test the model by imposing a set of "weak" lower and upper bounds and then "stronger" ones.

(a) $\mathbf{l}_{\mathrm{B}}=(0.1,0.1,0.1,0.1,0.1,0)^{\prime}, \mathbf{u}_{\mathrm{B}}=(0.3,0.3,0.3,0.3,0.3,1)^{\prime}$.

(b) $\mathbf{l}_{\mathrm{B}}=(0.1,0.02,0.02,0.1,0.02,0)^{\prime}, \mathbf{u}_{\mathrm{B}}=(0.5,0.1,0.1,0.5,0.1,1)^{\prime}$.

Assume the investors have an initial wealth $W_{0}=1$ and invest it to purchase the market portfolio $\mathbf{r}^{\prime} \mathbf{w}$. On $t=0$, the investment is rolled-forward monthly. The final value of wealth is computed recursively by the following algorithm (A1) 9

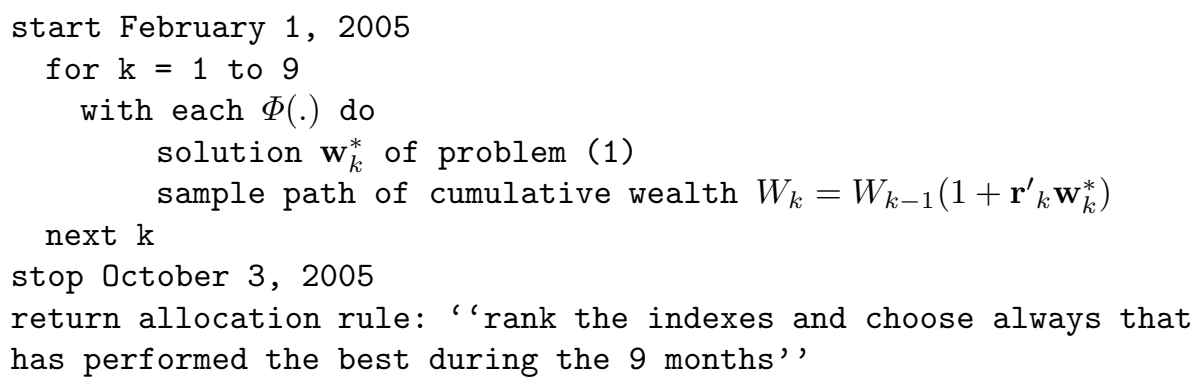

The optimal decay factor $\lambda^{*}$ for the returns is computed by the following algorithm (A2) 10

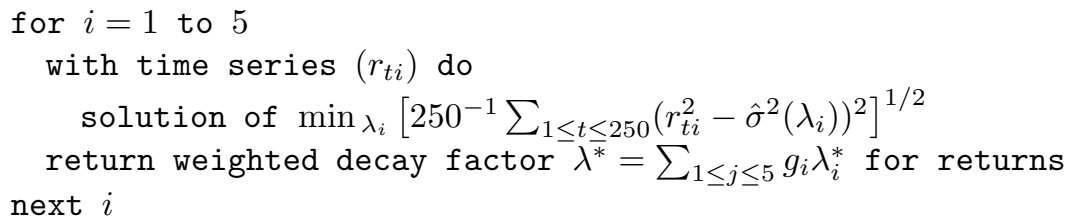

Algorithm (A1) iterates algorithm (A2) 11 Results in table 1 show the best forecast of investment performance being with stable ratio, during the last 9 months. With the Farinelli-Tibiletti ratio the investment performance is ranked either $9^{\text {th }}$ or $10^{\text {th }}$ based on agent's feelings towards overperformance above the risk-free rate. If the invest-

\footnotetext{
${ }^{8}$ In this study period all the stock indexes are very volatile, have a positive correlation, and weak stochastic dependence over time.

${ }^{9}$ The optimization problem (1) gives raise to the optimal excess weights $\mathbf{w}^{*}:=\mathbf{w}_{\mathbf{b}}+\mathbf{w}_{\mathbf{p}}{ }^{*}$. Clearly, we have implemented this problem by imposing $\mathbf{w}_{\mathbf{b}}=\mathbf{0}$ (no cash position).

${ }^{10}$ The weights are given by $g_{i}=\theta_{i}^{-1} / \sum_{1 \leq i \leq 5} \theta_{i}^{-1}$, where $\theta_{i}=\lambda_{i}^{*} / \sum_{1 \leq i \leq 5} \lambda_{i}^{*}$.

${ }^{11}$ The authors have developed an algorithm coded in MATLAB ${ }^{\complement}$. Moreover, to estimate the alpha index of return stability they used the Stable software by John P. Nolan [11]. A forthcoming version of the software will embed the quantile estimation algorithm coded by J. H. McCulloch [10]. All the values are rounded to the first fourteen decimals.
} 
Table 1. Values of final wealth $W_{9}$

\begin{tabular}{l|c}
\hline \hline Sharpe & 1.06694629842668 \\
Minimax & 1.06811617301054 \\
MAD & 1.05841393259069 \\
Gini & 1.05125992095915 \\
Sortino-Satchell & 1.01840417326684 \\
VaR & 1.07558191178067 \\
STARR & 1.07415420051962 \\
Rachev & 1.06304804004463 \\
Generalized Rachev & 1.04271604120210 \\
Farinelli-Tibiletti & $1.04342155326597(1.03617932688779)$ \\
Stable & 1.08034901565430 \\
\hline
\end{tabular}

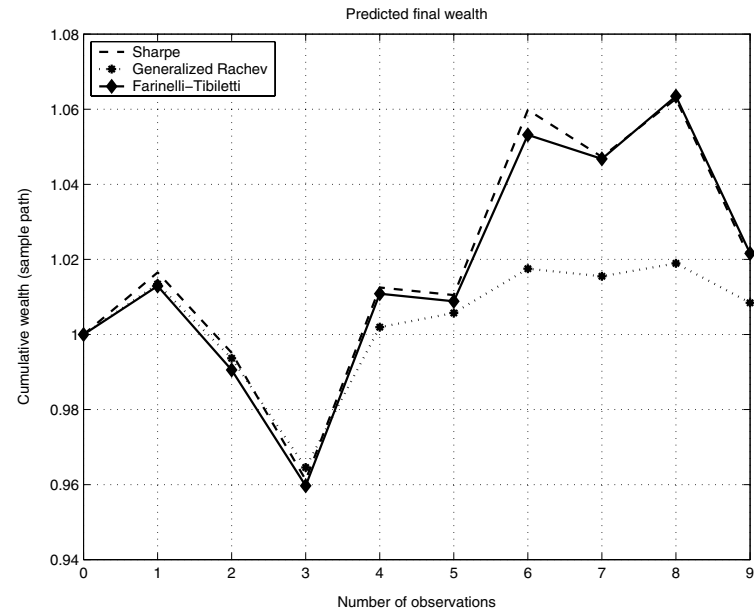

Fig. 1. Comparison among different values of final wealth computed with Sharpe ratio (dashed line), with Farinelli-Tibiletti ratio (solid line), and with Generalized Rachev ratio (dotted line)

ment management provides information about the history of stock prices, the investors can modify their preferences requiring alternative portfolio restrictions according to the empirical features of past returns. Case (b) corresponds to claim a greater investment in S\&P500 and FSTE than in the remaining stock indexes, since these two assets have showed "better" skewness and kurtosis features during the past 258 months than the other assets as it can be seen in table 2. The values of final wealth with those new portfolio constrains considered in (A 1) are listed in table 3 We note the Farinelli-Tibiletti ratio with left order $p=2$ and right order $q=0.5$ having a superior forecasting power than the Sharpe ratio, since this parameter setting better fits the agent's willingness to a performance over the benchmark, but a lower risk.

The Sharpe ratio has a low forecasting ability, while the Sortino-Satchell ratio provides a more robust performance evaluation. Nevertheless, indexes based on one-sided 
Table 2. Summary statistics

\begin{tabular}{l|cc}
\hline & \multicolumn{2}{|c}{ Coef. of skewness Coef. of kurtosis } \\
\hline \hline S\&P500 & -1.0452 & 7.0024 \\
Dow Jones & -1.2717 & 9.1074 \\
NASDAQ & -1.1771 & 8.1169 \\
FTSE & -0.9259 & 5.5730 \\
NIKKEI & -0.3526 & 3.6497 \\
\hline
\end{tabular}

Table 3. Values of final wealth $W_{9}$

\begin{tabular}{l|c}
\hline \hline Sharpe & 1.02012809289746 \\
Minimax & 1.03261850863065 \\
MAD & 1.02731027560518 \\
Gini & 1.01821753891104 \\
Sortino-Satchell & 1.00079846908146 \\
VaR & 1.02847451885572 \\
STARR & 1.03561700766895 \\
Rachev & 1.00479358219820 \\
Generalized Rachev & 1.00844935672914 \\
Farinelli-Tibiletti & $1.02158214310971(1.02018870912263)$ \\
Stable & 1.02914075668800 \\
\hline
\end{tabular}

Table 4. Values of periodic ( 9 months) wealth, $p=2, q=0.5$. Different results in parentheses (rows 15th and 16th, $p=0.5, q=2$ )

\begin{tabular}{l|c}
\hline \hline Generalized Rachev & 1.10292296179689 \\
Generalized Rachev & 0.98606688783133 \\
Gini & 1.13870280665459 \\
MAD & 1.27064360179623 \\
Sortino-Satchell & 1.16576029275820 \\
Rachev 1 & 1.22929409983253 \\
Rachev 1 & 1.24671887971556 \\
Sortino-Satchell & 1.14971024283881 \\
Rachev 2 & 1.16075620538274 \\
Sortino-Satchell & 1.35023369205268 \\
Generalized Rachev & 0.90595224732194 \\
Stable & 1.02587651156297 \\
Generalized Rachev & 0.70932459615394 \\
Sortino-Satchell & 1.27960286499482 \\
Rachev 2 & 1.19949421938987 (Farinelli-Tibiletti: 1.20454081121982) \\
Farinelli-Tibiletti & 1.08879861877932 (Sortino-Satchell: 1.08537313315757) \\
Farinelli-Tibiletti & 1.02346807285117 \\
\hline
\end{tabular}

variability measures such as the Generalized Rachev ratio and the Farinelli-Tibiletti ratio have a degree of flexibility in the measurement of portfolio overperformance. 


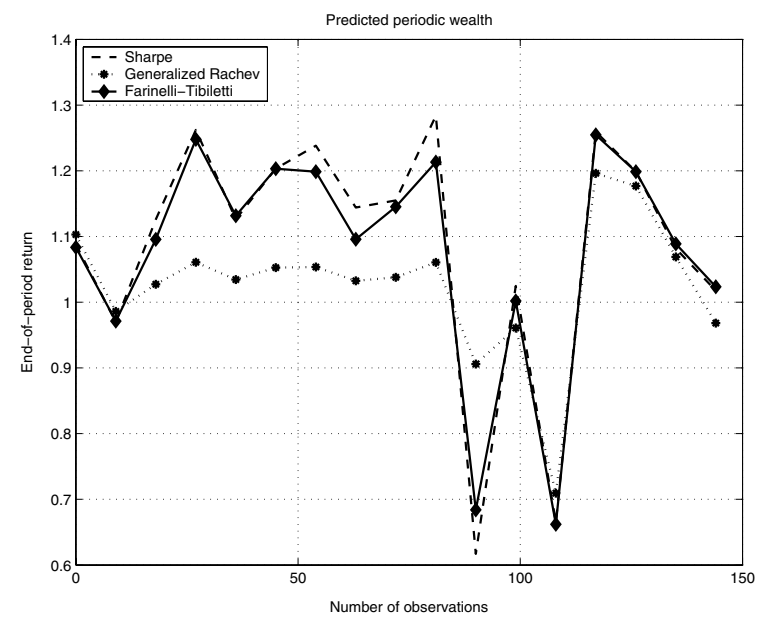

Fig. 2. Comparison among different values of periodic wealth computed with Sharpe ratio (dashed line), with Farinelli-Tibiletti ratio (solid line), and with Generalized Rachev ratio (dotted line)

Evaluating the portfolio's performance with respect to a benchmark additionally requires an out-of-sample analysis. We employ two moving windows: within the first (length 1 month) the final values of wealth (for each indexes) are calculated; within the second (length 9 months) the robustness of investment ranking for all the ratios involved in the optimization procedure is tested. The robustness check algorithm follows:

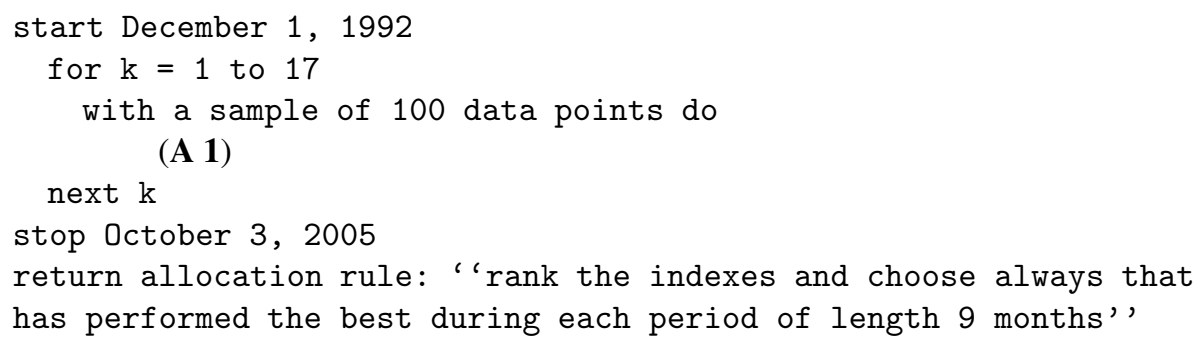

Results from robustness check algorithm are shown in table 4 in the case (b) 12

\section{Conclusion}

In this paper we solve a portfolio optimization problem comparing eleven performance indexes recently proposed by the literature, studying their behavior on wealth investment. Indexes based on one-sided variability measures are a flexible tool for modelling the agent's beliefs about either underperformance or overperformance a given benchmark. Further empirical tests will be the subject of future research. Particularly, we need

\footnotetext{
${ }^{12}$ We employ different parameter setting for the Rachev ratio: $\alpha=\beta=0.05$ (Rachev 2); $\alpha=0.01$ and $\beta=0.5$ (Rachev 3).
} 
to investigate the influence of the forecasting model for the expected returns, since from the mean-variance framework it is well known that optimal allocation depend in a very sensitive way on the expected returns.

\section{References}

1. Biglova, A., Huber, I., Ortobelli, S., Rachev, S.T.: Optimal Portfolio Selection and Risk Management: A Comparison Between the Stable Paretian Approach and the Gaussian One. In: Rachev, S.T. (ed.): Handbook on Computational and Numerical Methods in Finance. Birkhäuser, Boston (2004) 197-252

2. Biglova, A., Ortobelli, S., Rachev, S.T., Stoyanov, S.: Different Approaches to Risk Estimation in Portfolio Theory. Journal of Portfolio Management (2004) Fall, 103-112

3. Farinelli, S., Tibiletti, L.: Sharpe Thinking in Asset Ranking with One-Sided Measures. European Journal of Operational Research (2005) 5, forthcoming

4. Farinelli, S., Tibiletti, L.: Upside and Downside Risk with a Benchmark. Atlantic Economic Journal, Anthology Section, (2003) 31(4), December, 387

5. Farinelli, S., Tibiletti, L.: Sharpe Thinking with Asymmetrical Preferences. Technical Report, presented at European Bond Commission (2003) Winter Meeting, Frankfurt

6. Favre, L., Galeano, J.A.: Mean-Modified Value at Risk Optimization with Hedge Funds. The Journal of Alternative Investment (2002) 5, Fall

7. Huber, I., Ortobelli, S., Rachev, S.T., Schwartz, E.: Portfolio Choice Theory with NonGaussian Distributed Returns. In: Rachev, S.T. (ed.): Handbook of Heavy Tailed Distribution in Finance. Elsevier, Amsterdam (2003) 547-594

8. Konno, H., Yamazaki, H.: Mean-Absolute Deviation Portfolio Optimization Model and its Application to Tokyo Stock Market. Management Science (1991) 37, 519-531

9. Martin, D., Rachev, S.T., Siboulet, F.: Phi-Alpha Optimal Portfolios and Extreme Risk Management. Wilmott Magazine of Finance (2003) November, 70-83

10. McCulloch, J.H.: Simple Consistent Estimators of Stable Distribution Parameters. Commun. Statist. Simulation (1986) 15, 1109-1136

11. Nolan, J.P.: Numerical Approximations of Stable Densities and Distribution Functions. Commun. Statist. Stochastic Models (1997) 13, 759-774

12. Pedersen, C.S., Satchell, S.E.: On the Foundation of Performance Measures under Asymmetric Returns. Quantitative Finance (2003)

13. Shalit, H., Yitzhaki, S.: Mean-Gini, Portfolio Theory, and the Pricing of Risky Assets. Journal of Finance (1984) 39, 1449-1468

14. Sortino, F.A., van der Meer, R.: Downside Risk. Journal of Portfolio Management (1991) 17(4), 27-32

15. Sortino, F.A.: Upside-Potential Ratios Vary by Investment Style. Pensions and Investment (2000) 28, 30-35

16. Yitzhaki, S.: Stochastic Dominance, Mean Variance and Gini's Mean Difference. American Economic Review (1982) 72, 178-185

17. Young, M.R.: A MiniMax Portfolio Selection Rule with Linear Programming Solution. Management Science (1998) 44, 673-683 